\title{
SOME INEQUALITIES OF ČEBYŠEV TYPE FOR FUNCTIONS OF OPERATORS IN HILBERT SPACES
}

\author{
S. S. DRAGOMIR
}

\begin{abstract}
Some operator inequalities for synchronous functions that are related to the Čebyšev inequality for sequences of real numbers are given. Natural examples for pairs of functions that have the same monotonicity on an interval are presented as well.
\end{abstract}

\section{INTRODUCTION}

For $\mathbf{p}=\left(p_{1}, \ldots, p_{n}\right), \mathbf{a}=\left(a_{1}, \ldots, a_{n}\right)$ and $\mathbf{b}=\left(b_{1}, \ldots, b_{n}\right) n$-tuples of real numbers, consider the Čebyšev functional

$$
T_{n}(\mathbf{p} ; \mathbf{a}, \mathbf{b}):=P_{n} \sum_{i=1}^{n} p_{i} a_{i} b_{i}-\sum_{i=1}^{n} p_{i} a_{i} \cdot \sum_{i=1}^{n} p_{i} b_{i},
$$

where $P_{n}:=\sum_{i=1}^{n} p_{i}$.

In 1882-1883, Cebyšev [1] and [2] proved that, if $\mathbf{a}$ and $\mathbf{b}$ are monotonic in the same (opposite) sense and $\mathbf{p}$ is nonnegative, then

$$
T_{n}(\mathbf{p} ; \mathbf{a}, \mathbf{b}) \geq(\leq) 0
$$

The inequality (1.2) was mentioned by Hardy, Littlewood and Polya in their book [7] in 1934 in the more general setting of synchronous sequences, i.e., if $\mathbf{a}, \mathbf{b}$ are synchronous (asynchronous), this means that

$$
\left(a_{i}-a_{j}\right)\left(b_{i}-b_{j}\right) \geq(\leq) 0 \text { for each } i, j \in\{1, \ldots, n\},
$$

then (1.2) holds true.

For general real weights p, Mitrinović and Pečarić has shown in [8] that the inequality (1.2) holds true if

$$
0 \leq P_{k} \leq P_{n} \text { for } k \in\{1, \ldots, n-1\},
$$

and $\mathbf{a}, \mathbf{b}$ are monotonic in the same (opposite) sense.

2010 Mathematics Subject Classification. 47A63, 47A99.

Key words and phrases. Selfadjoint bounded linear operators, functions of operators, power series. 
We say that the functions $f, g:[a, b] \longrightarrow \mathbb{R}$ are synchronous (asynchronous) on the interval $[a, b]$ if they satisfy the following condition:

$$
(f(t)-f(s))(g(t)-g(s)) \geq(\leq) 0 \text { for each } t, s \in[a, b] .
$$

It is obvious that, if $f, g$ are monotonic and have the same monotonicity on the interval $[a, b]$, then they are synchronous on $[a, b]$ while if they have opposite monotonicity, they are asynchronous.

For some extensions of the discrete Čebyšev inequality for synchronous (asynchronous) sequences of vectors in an inner product space, see [4] and [5].

The following result provides an inequality of Čebyšev type for functions of one selfadjoint operator:

Let $A$ be a selfadjoint operator on the Hilbert space $(H,\langle\cdot, \cdot\rangle)$ with $S p(A)$ $\subseteq[m, M]$ for some real numbers $m<M$. If $f, g:[m, M] \longrightarrow \mathbb{R}$ are continuous and synchronous (asynchronous) on $[m, M]$, then $[3]$

$$
\langle f(A) g(A) x, x\rangle \geq(\leq)\langle f(A) x, x\rangle \cdot\langle g(A) x, x\rangle
$$

for any $x \in H$ with $\|x\|=1$.

As a particular case of interest we notice that if $A$ is a positive selfadjoint operator on $H$, then

$$
\left\langle A^{p+q} x, x\right\rangle \geq\left\langle A^{p} x, x\right\rangle \cdot\left\langle A^{q} x, x\right\rangle
$$

for any $x \in H$ with $\|x\|=1$ and $p, q>0$.

Motivated by the above results, we introduce in the present paper the concept of operator synchronous (asynchronous) functions and provide some fundamental Čebyšev type inequalities. Applications for some elementary functions of interest are also provided.

\section{OPERATOR SYNCHRONOUS FUNCTIONS}

It is known, see for instance [9, p. 356-358], that if $A$ and $B$ are two commuting bounded selfadjoint operators on the complex Hilbert space $H$, then there exists a bounded selfadjoint operator $S$ on $H$ and two bounded functions $\varphi$ and $\psi$ such that $A=\varphi(S)$ and $B=\psi(S)$. Moreover, if $\left\{E_{\lambda}\right\}$ is the spectral family over the closed interval $[0,1]$ for the selfadjoint operator $S$, then $S=\int_{0-}^{1} \lambda d E_{\lambda}$, where the integral is taken in the Riemann-Stieltjes sense, the functions $\varphi$ and $\psi$ are summable with respect with $\left\{E_{\lambda}\right\}$ on $[0,1]$ and

$$
A=\varphi(S)=\int_{0-}^{1} \varphi(\lambda) d E_{\lambda} \text { and } B=\psi(S)=\int_{0-}^{1} \psi(\lambda) d E_{\lambda} .
$$

Now, if $A$ and $B$ are as above with $S p(A), S p(B) \subseteq J$ an interval of real numbers, then for any continuous functions $f, g: J \rightarrow \mathbb{C}$ we have the 
representations

$$
f(A)=\int_{0-}^{1}(f \circ \varphi)(\lambda) d E_{\lambda} \text { and } g(B)=\int_{0-}^{1}(g \circ \psi)(\lambda) d E_{\lambda} .
$$

Definition 1. We say that the continuous functions $f, g: J \rightarrow \mathbb{R}$ are operator synchronous (asynchronous) on $J$, if for any $A$ and $B$ two commuting bounded selfadjoint operators on the complex Hilbert space $H$ with $S p(A), S p(B) \subseteq J$ we have

$$
(f(A)-f(B))(g(A)-g(B)) \geq(\leq) 0
$$

in the operator order.

In what follows, unless specified, $H$ will be a complex Hilbert space.

Theorem 1. The continuous functions $f, g: J \rightarrow \mathbb{R}$ are synchronous (asynchronous) on $J$ if and only if they are operator synchronous (asynchronous) on $J$.

Proof. ( $\Longrightarrow$ ) Let $A$ and $B$ two commuting bounded selfadjoint operators on the Hilbert space $H$ with $S p(A), S p(B) \subseteq J$. Then we have the representations (2.1) and (2.2).

Now, if $f, g: J \rightarrow \mathbb{R}$ are synchronous on $J$, then

$$
0 \leq(f(\varphi(\lambda))-f(\psi(\mu)))(g(\varphi(\lambda))-g(\psi(\mu)))
$$

for $\lambda, \mu \in[0,1]$ where the functions $\varphi$ and $\psi$ are the functions from (2.1).

Therefore

$$
\begin{aligned}
0 \leq & \int_{0-}^{1} \int_{0-}^{1}(f(\varphi(\lambda))-f(\psi(\mu)))(g(\varphi(\lambda))-g(\psi(\mu))) d E_{\lambda} d E_{\mu} \\
= & \int_{0-}^{1} \int_{0-}^{1}[f(\varphi(\lambda)) g(\varphi(\lambda))+f(\psi(\mu)) g(\psi(\mu)) \\
& -g(\varphi(\lambda)) f(\psi(\mu))-f(\varphi(\lambda)) g(\psi(\mu))] d E_{\lambda} d E_{\mu} \\
= & \int_{0-}^{1} f(\varphi(\lambda)) g(\varphi(\lambda)) d E_{\lambda} \int_{0-}^{1} d E_{\mu} \\
& +\int_{0-}^{1} d E_{\lambda} \int_{0-}^{1} f(\psi(\mu)) g(\psi(\mu)) d E_{\mu} \\
& -\int_{0-}^{1} g(\varphi(\lambda)) d E_{\lambda} \int_{0-}^{1} f(\psi(\mu)) d E_{\mu} \\
& -\int_{0-}^{1} f(\varphi(\lambda)) d E_{\lambda} \int_{0-}^{1} g(\psi(\mu)) d E_{\mu} \\
= & f(A) g(A)+f(B) g(B)-g(A) f(B)-f(A) g(B) \\
= & (f(A)-f(B))(g(A)-g(B))
\end{aligned}
$$


since, obviously, by the commutativity of $A$ with $B$ we have $f(B) g(A)=$ $g(A) f(B)$.

$(\Longleftarrow)$ If the inequality $(2.3)$ holds for any $A$ and $B$ two commuting bounded selfadjoint operators on the Hilbert space $H$ with $S p(A), S p(B) \subseteq J$, then by choosing $A=s 1_{H}$ and $B=t 1_{H}$ with $s, t \in J$ we deduce that $(f(s)-f(t))(g(s)-g(t)) \geq(\leq) 0$ which concludes the proof.

Corollary 1. If the continuous functions $f, g: J \rightarrow \mathbb{R}$ have the same monotonicity on $J$ then for any $A$ and $B$ two commuting bounded selfadjoint operators on the Hilbert space $H$ with $S p(A), S p(B) \subseteq J$ we have

$$
f(A) g(A)+f(B) g(B) \geq g(A) f(B)+f(A) g(B)
$$

in the operator order.

Remark 1. We observe that the above inequality (2.5) can provide numerous inequalities of interest for two commuting selfadjoint operators.

For instance, if $A$ and $B$ are positive commuting operators on $H$ then for any $p, q>0$ we have

$$
A^{p+q}+B^{p+q} \geq B^{p} A^{q}+A^{p} B^{q} .
$$

If the commuting operators $A$ and $B$ are positive definite on $H$, then also

$$
A \ln (A)+B \ln (B) \geq B \ln (A)+A \ln (B) .
$$
then

Also, if $A$ and $B$ are commuting operators on $H$ with $0 \leq A, B \leq \frac{\pi}{2} 1_{H}$,

$$
\sin (A) \cos (A)+\sin (B) \cos (B) \leq \sin (B) \cos (A)+\sin (A) \cos (B) .
$$

Corollary 2. If the continuous functions $f, g: J \rightarrow \mathbb{R}$ are synchronous on $J$, then for any $A$ a bounded selfadjoint operator on the Hilbert space $H$ with $\operatorname{Sp}(A) \subseteq J$ we have

$$
\begin{aligned}
\langle f(A) g(A) y, y\rangle-\langle f(A) y, y\rangle\langle g(A) y, y\rangle \\
\geq[\langle g(A) y, y\rangle-g(\langle A x, x\rangle)][f(\langle A x, x\rangle)-\langle f(A) y, y\rangle]
\end{aligned}
$$

for any $x, y \in H$ with $\|x\|=\|y\|=1$.

In particular, we have

$$
\begin{aligned}
\langle f(A) g(A) & x, x\rangle-\langle f(A) x, x\rangle\langle g(A) x, x\rangle \\
& \geq[\langle g(A) x, x\rangle-g(\langle A x, x\rangle)][f(\langle A x, x\rangle)-\langle f(A) x, x\rangle]
\end{aligned}
$$

for any $x \in H$ with $\|x\|=1$. 
Proof. Since $f, g: J \rightarrow \mathbb{R}$ are operator synchronous on $J$, then by choosing $B=\langle A x, x\rangle 1_{H}$ with a given $x \in H$ with $\|x\|=1$, we have in the operator order the inequality

$$
\begin{aligned}
f(A) g(A)+f(\langle A x, x\rangle) g & (\langle A x, x\rangle) 1_{H} \\
& \geq f(\langle A x, x\rangle) g(A)+g(\langle A x, x\rangle) f(A) .
\end{aligned}
$$

If we take this inequality for vectors $y \in H$ with $\|y\|=1$, then we get

$$
\begin{aligned}
\langle f(A) g(A) y, y\rangle & +f(\langle A x, x\rangle) g(\langle A x, x\rangle) \\
& \geq f(\langle A x, x\rangle)\langle g(A) y, y\rangle+g(\langle A x, x\rangle)\langle f(A) y, y\rangle
\end{aligned}
$$

for any $x, y \in H$ with $\|x\|=\|y\|=1$.

This inequality is equivalent with

$$
\begin{aligned}
\langle f(A) g(A) & y, y\rangle-\langle f(A) y, y\rangle\langle g(A) y, y\rangle \\
& \geq f(\langle A x, x\rangle)\langle g(A) y, y\rangle+g(\langle A x, x\rangle)\langle f(A) y, y\rangle \\
& -f(\langle A x, x\rangle) g(\langle A x, x\rangle)-\langle f(A) y, y\rangle\langle g(A) y, y\rangle \\
& =[\langle g(A) y, y\rangle-g(\langle A x, x\rangle)][f(\langle A x, x\rangle)-\langle f(A) y, y\rangle]
\end{aligned}
$$

for any $x, y \in H$ with $\|x\|=\|y\|=1$.

Remark 2. The inequality (2.9) was obtained in a different way in [3] where also has been noted that if the continuous functions $f, g: J \rightarrow \mathbb{R}$ are synchronous on $J$ and one of them is convex while the other is concave, then we have the improvement of the inequality (1.5)

$$
\begin{aligned}
& \langle f(A) g(A) x, x\rangle-\langle f(A) x, x\rangle\langle g(A) x, x\rangle \\
& \quad \geq[\langle g(A) x, x\rangle-g(\langle A x, x\rangle)][f(\langle A x, x\rangle)-\langle f(A) x, x\rangle] \geq 0,
\end{aligned}
$$

for any $x \in H$ with $\|x\|=1$.

For some particular examples of interest see [3].

Theorem 2. Assume that the continuous functions $f, g: J \rightarrow \mathbb{R}$ are synchronous (asynchronous) on J. If $\left(A_{1}, \ldots, A_{n}\right)$ and $\left(B_{1}, \ldots, B_{n}\right)$ are two $n$-tuples of selfadjoint operators with the spectra in $J$ and such that $A_{k}$ commutes with $B_{j}$ for any $k, j \in\{1, \ldots, n\}$, then for any nonnegative $n$-tuples of real numbers $\left(p_{1}, \ldots, p_{n}\right)$ and $\left(q_{1}, \ldots, q_{n}\right)$ we have

$$
\begin{aligned}
Q_{n} \sum_{k=1}^{n} p_{k} f\left(A_{k}\right) g\left(A_{k}\right)+P_{n} \sum_{j=1}^{n} q_{j} f\left(B_{j}\right) g\left(B_{j}\right) \\
\quad \geq(\leq) \sum_{k=1}^{n} p_{k} g\left(A_{k}\right) \sum_{j=1}^{n} q_{j} f\left(B_{j}\right)+\sum_{k=1}^{n} p_{k} f\left(A_{k}\right) \sum_{j=1}^{n} q_{j} g\left(B_{j}\right),
\end{aligned}
$$


where $P_{n}, Q_{n}>0$.

Proof. Since $f, g: J \rightarrow \mathbb{R}$ are operator synchronous (asynchronous) on $J$ and $A_{k}$ commutes with $B_{j}$ for any $k, j \in\{1, \ldots, n\}$, then we have

$$
f\left(A_{k}\right) g\left(A_{k}\right)+f\left(B_{j}\right) g\left(B_{j}\right) \geq(\leq) g\left(A_{k}\right) f\left(B_{j}\right)+f\left(A_{k}\right) g\left(B_{j}\right)
$$

for any $k, j \in\{1, \ldots, n\}$.

Now, if we multiply the inequality (2.15) with $p_{k}$ and $q_{j}$ and sum over $k$ and $j$ from 1 to $n$ we deduce the desired result (2.14).

Remark 3. We observe that, in general, it is not necessary for the terms of the $n$-tuples $\left(A_{1}, \ldots, A_{n}\right)$ two commute between them. The same applies for $\left(B_{1}, \ldots, B_{n}\right)$.

The following particular case provides a Čebyšev type inequality for synchronous (asynchronous) functions.

Corollary 3. Assume that the continuous functions $f, g: J \rightarrow \mathbb{R}$ are synchronous (asynchronous) on J. If $\left(A_{1}, \ldots, A_{n}\right)$ is an n-tuples of selfadjoint operators such that $A_{k}$ commutes with $A_{j}$ for any $k, j \in\{1, \ldots, n\}$, then for any nonnegative $n$-tuples of real numbers $\left(p_{1}, \ldots, p_{n}\right)$ we have

$$
P_{n} \sum_{k=1}^{n} p_{k} f\left(A_{k}\right) g\left(A_{k}\right) \geq(\leq) \sum_{k=1}^{n} p_{k} f\left(A_{k}\right) \sum_{k=1}^{n} p_{k} g\left(A_{k}\right) \text {. }
$$

Remark 4. If $\left(A_{1}, \ldots, A_{n}\right)$ and $\left(B_{1}, \ldots, B_{n}\right)$ are two $n$-tuples of positive selfadjoint operators such that $A_{k}$ commutes with $B_{j}$ for any $k, j \in$ $\{1, \ldots, n\}$, then for any nonnegative $n$-tuples of real numbers $\left(p_{1}, \ldots, p_{n}\right)$ and $\left(q_{1}, \ldots, q_{n}\right)$ and for any $p, q>0$ we have

$$
\begin{aligned}
Q_{n} \sum_{k=1}^{n} p_{k} A_{k}^{p+q}+P_{n} \sum_{j=1}^{n} q_{j} & B_{j}^{p+q} \\
& \geq \sum_{k=1}^{n} p_{k} A_{k}^{q} \sum_{j=1}^{n} q_{j} B_{j}^{p}+\sum_{k=1}^{n} p_{k} A_{k}^{p} \sum_{j=1}^{n} q_{j} B_{j}^{q}
\end{aligned}
$$

where $P_{n}, Q_{n}>0$.

In particular, if $\left(A_{1}, \ldots, A_{n}\right)$ is an $n$-tuples of positive selfadjoint operators such that $A_{k}$ commutes with $A_{j}$ for any $k, j \in\{1, \ldots, n\}$, then for any nonnegative $n$-tuples of real numbers $\left(p_{1}, \ldots, p_{n}\right)$ we have

$$
P_{n} \sum_{k=1}^{n} p_{k} A_{k}^{p+q} \geq \sum_{k=1}^{n} p_{k} A_{k}^{p} \sum_{k=1}^{n} p_{k} A_{k}^{q}
$$

where $p, q>0$. 
If $\left(A_{1}, \ldots, A_{n}\right)$ is an $n$-tuples of positive definite selfadjoint operators such that $A_{k}$ commutes with $A_{j}$ for any $k, j \in\{1, \ldots, n\}$, then for any nonnegative $n$-tuples of real numbers $\left(p_{1}, \ldots, p_{n}\right)$ we have

$$
P_{n} \sum_{k=1}^{n} p_{k} A_{k} \ln A_{k} \geq \sum_{k=1}^{n} p_{k} A_{k} \sum_{k=1}^{n} p_{k} \ln A_{k} .
$$

The above inequality (2.17) may be used to prove the following result for functions of operators expressed by power series with nonnegative coefficients.

Theorem 3. Let $f(z)=\sum_{n=0}^{\infty} a_{n} z^{n}$ and $g(z)=\sum_{n=0}^{\infty} b_{n} z^{n}$ be two power series with nonnegative coefficients and convergent on the open disk $D(0, R)$ with $R>0$. If $A$ and $B$ are commuting positive operators and $p, q, t, s>0$ such that $t\|A\|^{p}, t\|A\|^{q}, t\|A\|^{p+q}<R$ and $s\|B\|^{p}, s\|B\|^{q}, s\|B\|^{p+q}<R$, then we have the inequality

$$
g(s) f\left(t A^{p+q}\right)+f(t) g\left(s B^{p+q}\right) \geq f\left(t A^{q}\right) g\left(s B^{p}\right)+f\left(t A^{p}\right) g\left(s B^{q}\right) .
$$

Proof. Since $A$ and $B$ are commuting positive operators, then $A_{k}:=A^{k}$ commutes with $B_{j}:=B^{j}$ for any $k, j \in\{0, \ldots, n\}$ and writing the inequality (2.17) for $p_{k}:=a_{k} t^{k}$ and $q_{j}:=b_{j} s^{j}$ where $k, j \in\{0, \ldots, n\}$, we have

$$
\begin{aligned}
\sum_{j=0}^{n} b_{j} s^{j} \sum_{k=0}^{n} a_{k} t^{k}\left(A^{p+q}\right)^{k}+\sum_{k=0}^{n} a_{k} t^{k} \sum_{j=0}^{n} b_{j} s^{j}\left(B^{p+q}\right)^{j} \\
\quad \geq \sum_{k=0}^{n} a_{k} t^{k}\left(A^{q}\right)^{k} \sum_{j=0}^{n} b_{j} s^{j}\left(B^{p}\right)^{j}+\sum_{k=0}^{n} a_{k} t^{k}\left(A^{p}\right)^{k} \sum_{j=0}^{n} b_{j} s^{j}\left(B^{q}\right)^{j}
\end{aligned}
$$

for any $n \in \mathbb{N}$.

Since all the series whose partial sums are involved in the inequality (2.21) are convergent, then by taking $n \rightarrow \infty$ in (2.21) we deduce the desired result (2.20).

Remark 5. We observe that the inequality (2.20) is an extension of the power inequality (2.6).

For some similar results, see the recent paper [6].

Example 1. 1. If $A$ and $B$ are commuting positive operators with $A, B<$ $1_{H}$, then we have the inequality

$$
\begin{aligned}
& \ln (1-s)^{-1}\left(1_{H}-t A^{p+q}\right)^{-1}+(1-t)^{-1} \ln \left(1_{H}-s B^{p+q}\right)^{-1} \\
& \quad \geq\left(1_{H}-t A^{q}\right)^{-1} \ln \left(1_{H}-s B^{p}\right)+\left(1_{H}-t A^{p}\right)^{-1} \ln \left(1_{H}-s B^{q}\right)^{-1}
\end{aligned}
$$

for any $s, t \in(0,1)$. 
2. If $A$ and $B$ are commuting positive operators, then we have the inequality

$$
\begin{aligned}
& \sinh (s) \cosh \left(t A^{p+q}\right)+\cosh (t) \sinh \left(s B^{p+q}\right) \\
& \geq \cosh \left(t A^{q}\right) \sinh \left(s B^{p}\right)+\cosh \left(t A^{p}\right) \sinh \left(s B^{q}\right)
\end{aligned}
$$

for any $s, t \in \mathbb{R}$.

We can consider the following functional

$$
\mathcal{C}_{(f, g)}(A, B):=f(A) g(A)+f(B) g(B)-g(A) f(B)-f(A) g(B)
$$

defined for the pair of continuous functions $(f, g)$ and the pair of commuting selfadjoint operators $(A, B)$ with $S p(A), S p(B) \subseteq J$, a given interval of $\mathbb{R}$.

Now, we can prove the following vector inequality:

Theorem 4. Let $(f, g)$ be continuous and synchronous functions on the interval $J$. Then for any pair of commuting selfadjoint operators $(A, B)$ with $S p(A), S p(B) \subseteq J$ and for any $x \in H$ with $\|x\|=1$, we have the inequality

$$
\left\langle\mathcal{C}_{(f, g)}(A, B) x, x\right\rangle \geq \max \left\{K_{1}, K_{2}, K_{3}\right\} \geq 0
$$

where

$$
K_{1}:=\left|\left\langle\mathcal{C}_{(|f|, g)}(A, B) x, x\right\rangle\right|, K_{2}:=\left|\left\langle\mathcal{C}_{(f,|g|)}(A, B) x, x\right\rangle\right|
$$

and

$$
K_{3}:=\left|\left\langle\mathcal{C}_{(|f|,|g|)}(A, B) x, x\right\rangle\right| .
$$

Proof. Let $x \in H$ with $\|x\|=1$. Utilising the identity (2.4) we have

$$
\begin{array}{r}
\int_{0-}^{1} \int_{0-}^{1}(f(\varphi(\lambda))-f(\psi(\mu)))(g(\varphi(\lambda))-g(\psi(\mu))) d\left\langle E_{\lambda} x, x\right\rangle d\left\langle E_{\mu} x, x\right\rangle \\
=\langle(f(A)-f(B))(g(A)-g(B)) x, x\rangle, \quad(2.26)
\end{array}
$$

where the function $h(\lambda):=\left\langle E_{\lambda} x, x\right\rangle$ and $m(\mu):=\left\langle E_{\mu} x, x\right\rangle$ are right continuous and monotonic nondecreasing on $[0,1]$ and the integral is taken in the Riemann-Stieltjes sense.

Since the functions $(f, g)$ are synchronous on $J$ then

$$
\begin{aligned}
& (f(\varphi(\lambda))-f(\psi(\mu)))(g(\varphi(\lambda))-g(\psi(\mu))) \\
& =|(f(\varphi(\lambda))-f(\psi(\mu)))(g(\varphi(\lambda))-g(\psi(\mu)))| \\
& =|f(\varphi(\lambda))-f(\psi(\mu))||g(\varphi(\lambda))-g(\psi(\mu))| \\
& \geq|| f(\varphi(\lambda))|-| f(\psi(\mu))|||g(\varphi(\lambda))-g(\psi(\mu))| \\
& =|(|f(\varphi(\lambda))|-|f(\psi(\mu))|)(g(\varphi(\lambda))-g(\psi(\mu)))|
\end{aligned}
$$

for any $\lambda, \mu \in[0,1]$. 
Integrating this inequality over the monotonic integrators $h(\lambda)$ and $m(\mu)$ we get

$$
\begin{gathered}
\int_{0-}^{1} \int_{0-}^{1}(f(\varphi(\lambda))-f(\psi(\mu)))(g(\varphi(\lambda))-g(\psi(\mu))) d\left\langle E_{\lambda} x, x\right\rangle d\left\langle E_{\mu} x, x\right\rangle \\
\geq \int_{0-}^{1} \int_{0-}^{1} \mid(|f(\varphi(\lambda))|-|f(\psi(\mu))|)(g(\varphi(\lambda)) \\
\geq\left|\int_{0-}^{1} \int_{0-}^{1}(|f(\varphi(\lambda))|-|f(\psi(\mu))|)(g(\varphi(\lambda)))\right| d\left\langle E_{\lambda} x, x\right\rangle d\left\langle E_{\mu} x, x\right\rangle \\
-g(\psi(\mu))) d\left\langle E_{\lambda} x, x\right\rangle d\left\langle E_{\mu} x, x\right\rangle \mid \\
=|\langle(|f(A)|-|f(B)|)(g(A)-g(B)) x, x\rangle|
\end{gathered}
$$

for any $x \in H$ with $\|x\|=1$.

The last equality above followed by the identity (2.26) applied for the functions $|f|$ and $g$ that are continuous on $J$.

This proves the inequality for $K_{1}$.

Similar inequalities can be obtained for $K_{2}$ and $K_{3}$. The details are however left to the reader.

Remark 6. If we apply the above result for the synchronous functions $f(t)=t^{p}$ with $p>0$ and $g(t)=\ln t$, then we get for any commuting positive definite operators $A$ and $B$ the inequality

$$
\begin{aligned}
& \left\langle\left(A^{p} \ln A+B^{p} \ln B-B^{p} \ln A-A^{p} \ln B\right) x, x\right\rangle \\
& \quad \geq\left|\left\langle\left(A^{p}|\ln A|+B^{p}|\ln B|-B^{p}|\ln A|-A^{p}|\ln B|\right) x, x\right\rangle\right|
\end{aligned}
$$

for any $x \in H$ with $\|x\|=1$.

If we take the synchronous functions $f(t)=t^{2 k+1}, g(t)=t^{2 m+1}$ with $k, m$ natural umbers, then we have for any commuting selfadjoint operators $A$ and $B$ the inequality

$$
\begin{aligned}
& \left\langle\left(A^{2 k+2 m+2}+B^{2 k+2 m+2}-A^{2 k+1} B^{2 m+1}-A^{2 m+1} B^{2 k+1}\right) x, x\right\rangle \\
\geq & \left|\left\langle\left(|A|^{2 k+2 m+2}+|B|^{2 k+2 m+2}-|A|^{2 k+1}|B|^{2 m+1}-|A|^{2 m+1}|B|^{2 k+1}\right) x, x\right\rangle\right|
\end{aligned}
$$

for any $x \in H$ with $\|x\|=1$.

The following integral inequality also holds:

Theorem 5. Let $(f, g)$ be continuous and synchronous functions on the interval $[m, M]$ and two integrable weights $w, v:[0,1] \rightarrow[0, \infty)$. Then for 
any pair of commuting selfadjoint operators $(A, B)$ with $S p(A), S p(B) \subseteq$ $[m, M]$ we have

$$
\begin{aligned}
& \int_{0}^{1} v(t) d t \int_{0}^{1} w(t) f(t A+(1-t) B) g(t A+(1-t) B) d t \\
& +\int_{0}^{1} w(t) d t \int_{0}^{1} v(t) f(t A+(1-t) B) g(t A+(1-t) B) d t \\
& \geq \int_{0}^{1} v(t) f(t A+(1-t) B) d t \int_{0}^{1} w(t) g(t A+(1-t) B) d t \\
& +\int_{0}^{1} w(t) f(t A+(1-t) B) d t \int_{0}^{1} v(t) g(t A+(1-t) B) d t
\end{aligned}
$$

and, in particular,

$$
\begin{aligned}
& \int_{0}^{1} w(t) d t \int_{0}^{1} w(t) f(t A+(1-t) B) g(t A+(1-t) B) d t \\
& \geq \int_{0}^{1} w(t) f(t A+(1-t) B) d t \int_{0}^{1} w(t) g(t A+(1-t) B) d t .
\end{aligned}
$$

Proof. Since $A$ and $B$ are commuting selfadjoint operators, then for any $t, s \in[0,1], t A+(1-t) B$ and $s A+(1-s) B$ are commuting selfadjoint operators.

Since $(f, g)$ are continuous and synchronous functions on the interval $[m, M]$, then

$$
\begin{aligned}
f(t A+(1-t) B) & g(t A+(1-t) B) \\
+ & f(s A+(1-s) B) g(s A+(1-s) B) \\
\geq f(s A+ & (1-s) B) g(t A+(1-t) B) \\
& \quad+f(t A+(1-t) B) g(s A+(1-s) B)
\end{aligned}
$$

for any $t, s \in[0,1]$.

Now, if we multiply (2.31) with the nonnegative quantities $w(t) v(s)$ and integrate over $t$ and $s$ on $[0,1]$, we deduce the desired result (2.30).

Corollary 4. With the assumptions from Theorem 5 for $(f, g)$ and $(A, B)$ we have the inequality

$$
\begin{aligned}
\int_{0}^{1} f(t A+(1-t) B) g(t A+(1-t) B) d t \\
\quad \geq \int_{0}^{1} f(t A+(1-t) B) d t \int_{0}^{1} g(t A+(1-t) B) d t .
\end{aligned}
$$


Remark 7. Assume that $A$ and $B$ are two commuting positive operators and such that $A-B$ is invertible. Then for $p>0$, by utilizing the representation (2.1) and Fubini's theorem, we have

$$
\begin{aligned}
\int_{0}^{1}((1-\lambda) A+\lambda B)^{p} d \lambda & =\int_{0}^{1}\left(\int_{0-}^{1}[(1-\lambda) \varphi(t)+\lambda \psi(t)]^{p} d E_{t}\right) d \lambda \\
& =\int_{0-}^{1}\left(\int_{0}^{1}[(1-\lambda) \varphi(t)+\lambda \psi(t)]^{p} d \lambda\right) d E_{t} \\
& =\frac{1}{p+1} \int_{0-}^{1} \frac{\varphi^{p+1}(t)-\psi^{p+1}(t)}{\varphi(t)-\psi(t)} d E_{t} \\
& =\frac{1}{p+1}(A-B)^{-1}\left(A^{p+1}-B^{p+1}\right) .
\end{aligned}
$$

Similarly, if $A$ and $B$ are two commuting positive definite operators and such that $A-B$ is invertible, then for $p \in(-\infty, 0) \backslash\{-1\}$ we also have

$$
\int_{0}^{1}((1-\lambda) A+\lambda B)^{p} d \lambda=\frac{1}{p+1}(A-B)^{-1}\left(A^{p+1}-B^{p+1}\right) .
$$

Also, if $A$ and $B$ are two commuting positive definite operators and such that $A-B$ is invertible, then

$$
\int_{0}^{1}((1-\lambda) A+\lambda B)^{-1} d \lambda=(A-B)^{-1}(\ln A-\ln B) .
$$

On applying the inequality (2.32) for the synchronous functions $f(t)=$ $t^{p}, g(t)=t^{q}$ with $p, q>0$, we get the inequality

$$
\begin{aligned}
& \frac{1}{p+q+1}(A-B)^{-1}\left(A^{p+q+1}-B^{p+q+1}\right) \\
& \quad \geq \frac{1}{p+1}(A-B)^{-1}\left(A^{p+1}-B^{p+1}\right) \cdot \frac{1}{q+1}(A-B)^{-1}\left(A^{q+1}-B^{q+1}\right),
\end{aligned}
$$

where $A$ and $B$ are commuting positive selfadjoint operators and $A-B$ is invertible.

Remark 8. In the case of real Hilbert spaces the concept of operator synchronous functions is equivalent with

$$
f(A) g(A)+f(B) g(B) \geq f(B) g(A)+f(A) g(B)
$$

where $A$ and $B$ can be noncommutative.

In that situation, we can get the inequality:

If $\left(A_{1}, \ldots, A_{n}\right)$ and $\left(B_{1}, \ldots, B_{n}\right)$ are two $n$-tuples of selfadjoint operators with the spectra in $J$ then for any nonnegative $n$-tuples of real numbers 
$\left(p_{1}, \ldots, p_{n}\right)$ and $\left(q_{1}, \ldots, q_{n}\right)$ and two continuous functions $f, g: J \rightarrow \mathbb{R}$ that are operator synchronous (asynchronous) on $J$ we have

$$
\begin{aligned}
Q_{n} \sum_{k=1}^{n} p_{k} f\left(A_{k}\right) g\left(A_{k}\right)+P_{n} \sum_{j=1}^{n} q_{j} f\left(B_{j}\right) g\left(B_{j}\right) \\
\quad \geq(\leq) \sum_{j=1}^{n} q_{j} f\left(B_{j}\right) \sum_{k=1}^{n} p_{k} g\left(A_{k}\right)+\sum_{k=1}^{n} p_{k} f\left(A_{k}\right) \sum_{j=1}^{n} q_{j} g\left(B_{j}\right)
\end{aligned}
$$

where $P_{n}, Q_{n}>0$.

In particular, we have

$$
P_{n} \sum_{k=1}^{n} p_{k} f\left(A_{k}\right) g\left(A_{k}\right) \geq(\leq) \sum_{k=1}^{n} p_{k} f\left(A_{k}\right) \sum_{k=1}^{n} p_{k} g\left(A_{k}\right)
$$

with no commutativity condition between the terms of $\left(A_{1}, \ldots, A_{n}\right)$.

Other similar results may be stated in the real case, however the details are left to the interested reader.

\section{Other ČEBYŠEv TYPE INEQUALITIES}

Let $(H,\langle.,\rangle$.$) be a complex Hilbert space.$

Definition 2. We say that two commuting selfadjoint operators $A$ and $B$ are power-synchronous if

$$
\left(A^{k}-A^{j}\right)\left(B^{k}-B^{j}\right) \geq 0
$$

for any $k, j \in \mathbb{N}$.

Theorem 6. Let $A$ and $B$ be two commuting selfadjoint operators. If either $0 \leq A \leq 1_{H}$ and $0 \leq B \leq 1_{H}$ or $A \geq 1_{H}$ and $B \geq 1_{H}$ then $A$ and $B$ are power-synchronous.

Proof. Utilising the representation (2.1) and (2.2), we have for any $k$ natural number

$$
A^{k}=\int_{0-}^{1} \varphi^{k}(\lambda) d E_{\lambda} \text { and } B^{k}=\int_{0-}^{1} \psi^{k}(\mu) d E_{\mu} .
$$

If $0 \leq A \leq 1_{H}$ and $0 \leq B \leq 1_{H}$ then the representing functions $\varphi$ and $\psi$ take the values in $[0,1]$ almost everywhere. 
We have for $k>j$

$$
\begin{aligned}
& \left(A^{k}-A^{j}\right)\left(B^{k}-B^{j}\right) \\
& =\int_{0-}^{1}\left(\varphi^{k}(\lambda)-\varphi^{j}(\lambda)\right) d E_{\lambda} \int_{0-}^{1}\left(\psi^{k}(\mu)-\psi^{j}(\mu)\right) d E_{\mu} \\
& =\int_{0-}^{1} \int_{0-}^{1}\left(\varphi^{k}(\lambda)-\varphi^{j}(\lambda)\right)\left(\psi^{k}(\mu)-\psi^{j}(\mu)\right) d E_{\lambda} d E_{\mu} \\
& =\int_{0-}^{1} \int_{0-}^{1} \varphi^{j}(\lambda) \psi^{j}(\mu)\left(\varphi^{k-j}(\lambda)-1\right)\left(\psi^{k-j}(\mu)-1\right) d E_{\lambda} d E_{\mu} \geq 0 .
\end{aligned}
$$

The same inequality holds if $k<j$. Therefore for any $k, j \in \mathbb{N}$ we have (3.1).

If $A \geq 1_{H}$ and $B \geq 1_{H}$ the proof goes likewise and the details are omitted.

Utilizing this concept we can state the following Čebyšev type inequality.

Theorem 7. Let $f(z)=\sum_{n=0}^{\infty} a_{n} z^{n}$ and be $g(z)=\sum_{n=0}^{\infty} b_{n} z^{n}$ be two functions defined by power series with nonnegative coefficients and convergent on the open disk $D(0, R) \subset \mathbb{C}, R>0$. If $A$ and $B$ are power-synchronous, $p, q$ two real numbers with $0 \leq p, q<R$ and such that

$$
a\|A B\|, q\|A B\|, p\|A\|, q\|B\|, q\|A\|, p\|B\|<R
$$

then we have the inequality

$$
g(q) f(p A B)+f(p) g(q A B) \geq f(p A) g(q B)+g(q A) f(p B) .
$$

Proof. Since $A$ and $B$ are power-synchronous, then we have

$$
A^{k} B^{k}+A^{j} B^{j} \geq A^{k} B^{j}+A^{j} B^{k}
$$

for any $k, j \in \mathbb{N}$.

Since $A$ and $B$ are commuting operators we have $A^{k} B^{k}=(A B)^{k}$ and $A^{j} B^{j}=(A B)^{j}$ for any $k, j \in \mathbb{N}$, then

$$
(A B)^{k}+(A B)^{j} \geq A^{k} B^{j}+A^{j} B^{k}
$$

for any $k, j \in \mathbb{N}$.

Now, if we multiply the inequality (3.4) by $a_{k} p^{k} b_{j} q^{j} \geq 0$ and sum over $k$ and $j$ from 0 to $m$ we get

$$
\begin{aligned}
& \sum_{j=0}^{m} b_{j} q^{j} \sum_{k=0}^{m} a_{k} p^{k}(A B)^{k}+\sum_{k=0}^{m} a_{k} p^{k} \sum_{j=0}^{m} b_{j} q^{j}(A B)^{j} \\
& \geq \sum_{k=0}^{m} a_{k} p^{k} A^{k} \sum_{j=0}^{m} b_{j} q^{j} B^{j}+\sum_{j=0}^{m} b_{j} q^{j} A^{j} \sum_{k=0}^{m} a_{k} p^{k} B^{k} .
\end{aligned}
$$


We observe that the series whose partial sums are involved in the inequality (3.5) are convergent, then by letting $m \rightarrow \infty$ in (3.5) we deduce the desired result (3.3).

A particular case of interest is when $g=f$.

Corollary 5. Let $f(z)=\sum_{n=0}^{\infty} a_{n} z^{n}$ be a function defined by power series with nonnegative coefficients and convergent on the open disk $D(0, R) \subset \mathbb{C}$, $R>0$. If $A$ and $B$ are power-synchronous, $p$ a real number with $0 \leq p<R$ and such that

$$
p\|A B\|, p\|A\|, p\|B\|<R
$$

then we have the inequality

$$
f(p) f(p A B) \geq f(p A) f(p B) .
$$

Example 2. 1. For the commuting operators $A$ and $B$, assume that $0 \leq$ $A<1_{H}$ and $0 \leq B<1_{H}$ and $p$ is a real number with $0 \leq p<1$. Then we have the inequalities

$$
\left(1_{H}-p A B\right)^{-1} \geq(1-p)\left(1_{H}-p A\right)^{-1}\left(1_{H}-p B\right)^{-1}
$$

and

$$
\ln (1-p)^{-1} \ln \left(1_{H}-p A B\right)^{-1} \geq \ln \left(1_{H}-p A\right)^{-1} \ln \left(1_{H}-p B\right)^{-1} .
$$

2. For the commuting operators $A$ and $B$, assume that either $0 \leq A \leq 1_{H}$ and $0 \leq B \leq 1_{H}$ or $A \geq 1_{H}$ and $B \geq 1_{H}$ and $p \geq 0$, then we have the inequalities

$$
\begin{aligned}
& \sinh (p) \sinh (p A B) \geq \sinh (p A) \sinh (p B), \\
& \cosh (p) \cosh (p A B) \geq \cosh (p A) \cosh (p B),
\end{aligned}
$$

and

$$
\exp (p) \exp (p A B) \geq \exp (p A) \exp (p B) .
$$

Acknowledgement. The author would like to thank Professor M. Uchiyama from Shimane University, Japan, for his important observation regarding the equivalence of usual synchronicity and operator synchronicity that played a key role above.

\section{REFERENCES}

[1] P. L. Čebyšev, O približennyh vyraženijah odnih integralov čerez drugie, Soobšcenija i protokoly zasedaniı̌ Matemmatičeskogo občestva pri Imperatorskom Har'kovskom Universitete, No. 2, (1882), 93-98; Polnoe sobranie sočinenǐ P. L. Čebyševa. MoskvaLeningrad, 1948a, 128-131. 
[2] P.L. Čebyšev, Ob odnom rjade, dostavljajušćem predel'nye veličiny integralov pri razloženii podintegral'nou funkcii na množeteli, Priloženi k 57 tomu Zapisok Imp. Akad. Nauk, No. 4; (1883) Polnoe sobranie sočinenǐ P. L. Čebyševa. Moskva-Leningrad, 1948b, 157-169.

[3] S. S. Dragomir, Čebyšev's type inequalities for functions of selfadjoint operators in Hilbert spaces, Linear Multilinear Algebra, 58 (7-8) (2010), 805-814.

[4] S. S. Dragomir and J. Sándor, The Chebyshev inequality in pre-Hilbertian spaces, I., Proc. of the Second Symposium of Mathematics and its Applications (Timişoara, 1987), 61-64, Res. Centre, Acad. SR Romania, Timişoara, 1988, MR1006000 (90k:46048).

[5] S. S. Dragomir, J. Pečarić and J. Sándor, The Chebyshev inequality in pre-Hilbertian spaces, II. Proceedings of the Third Symposium of Mathematics and its Applications (Timişoara, 1989), 75-78, Rom. Acad., Timişoara, 1990. MR1266442 (94m:46033)

[6] S. S. Dragomir and M. Uchiyama, Some inequalities for power series of two operators in Hilbert spaces, Tokyo J. Math. 36 (2) (2013), 483-498, Preprint RGMIA Res. Rep. Coll., 15 (2012), Article 30. [Online http://rgmia.org/v15.php].

[7] G. H. Hardy, J. E. Littlewood and G. Pólya, Inequalities, 1st Ed. and 2nd Ed. Cambridge University Press, Cambridge, (1934, 1952), England.

[8] D. S. Mitrinović and J. Pečarić, On an identity of D.Z. Djoković, Prilozi Mak. Akad. Nauk. Umj. (Skopje), 12 (1) (1991), 21-22.

[9] F. Riesz and B. Sz-Nagy, Functional Analysis, New York, Dover Publications, 1990.

(Received: December 10, 2013)

Mathematics

College of Engineering \& Science

Victoria University, PO Box 14428

Melbourne City, MC 8001

Australia

sever.dragomir@vu.edu.au

URL http://rgmia.org/dragomir

School of Comput. \& Applied Math. University of the Witwatersrand

Private Bag 3, Johannesburg 2050

South Africa 